\title{
NSAID-Induced Nephropathy
}

National Cancer Institute

\section{Source}

National Cancer Institute. NSAID-Induced Nephropathy. NCI Thesaurus. Code C123138.

Kidney damage resulting from exposure to non-steroidal anti-inflammatory drugs (NSAIDs). 